PROCEEDINGS OF THE

AMERICAN MATHEMATICAL SOCIETY

Volume 138, Number 3, March 2010, Pages 997-1010

S 0002-9939(09)10110-7

Article electronically published on October 21, 2009

\title{
FIBRATIONS AND FUNDAMENTAL GROUPS OF KÄHLER-WEYL MANIFOLDS
}

\author{
G. KOKAREV AND D. KOTSCHICK
}

(Communicated by Jon G. Wolfson)

\begin{abstract}
We extend the Siu-Beauville theorem to a certain class of compact Kähler-Weyl manifolds, proving that they fiber holomorphically over hyperbolic Riemannian surfaces whenever they satisfy the necessary topological hypotheses. As applications we obtain restrictions on the fundamental groups of such Kähler-Weyl manifolds, and we show that in certain cases they are in fact Kähler.
\end{abstract}

\section{INTRODUCTION}

There are many results concerning the topology of complex algebraic varieties and of compact Kähler manifolds that are proved using analytic methods such as Hodge theory and harmonic maps. These methods strongly depend on differentialgeometric features of Kähler manifolds that ultimately derive from the Kähler identities. Although the methods do not immediately extend, sometimes such results do generalize to non-Kähler compact complex surfaces using a case-by-case analysis appealing to the Enriques-Kodaira classification. It turns out that Kähler manifolds and complex surfaces are both special cases of complex manifolds supporting Kähler-Weyl structures, and some results that were previously known with disparate proofs in those two special cases can actually be proved uniformly for a certain class of Kähler-Weyl manifolds.

The notion of a Kähler-Weyl structure arises naturally in conformal geometry and goes back several decades to papers of Vaisman. We shall give a brief account of the basic definitions in Section 2 below, and we refer the reader to [7, [12] and the references cited therein for further details. The upshot is that the KählerWeyl condition is vacuous in complex dimensions one and two, just like the Kähler condition is vacuous in dimension one, and is equivalent to the locally conformally Kähler condition in higher dimensions.

Recently the first author extended some of the harmonic map techniques from Kähler geometry to the more general setting of Kähler-Weyl geometry; see [19]. This extension involves the study of pseudo-harmonic or Weyl harmonic maps from Kähler-Weyl domains endowed with so-called pluricanonical metrics. We recall the notion of a pluricanonical metric in Section 2 it covers a large class of examples and makes the Bochner technique applicable in the setting of Weyl harmonic maps.

Received by the editors November 12, 2008, and, in revised form, July 9, 2009.

2000 Mathematics Subject Classification. Primary 32J27, 32Q55, 53C55; Secondary 53C28, $53 \mathrm{C} 43,58 \mathrm{C} 10$. 
In this paper we use the techniques and results from [19] to prove and apply a generalization of the following theorem due to Siu and Beauville:

Theorem 1.1. For a compact Kähler manifold $M$ the following two statements are equivalent:

I. $M$ admits a surjective holomorphic map with connected fibers to a compact Riemann surface of genus $\geq 2$, and

II. $\pi_{1}(M)$ admits a surjective homomorphism to the fundamental group of a compact Riemann surface of genus $\geq 2$.

Obviously the first statement implies the second. The converse was first proved by Siu [26] using harmonic maps. It was later rediscovered by Beauville, whose proof is a sophisticated application of Hodge theory; see the appendix to [10. It is not hard to see, and is explained in [1, Chapter 2], that in the situation of Theorem 1.1 the two statements are also equivalent to the following:

III. $\pi_{1}(M)$ admits a surjective homomorphism to a non-abelian free group.

A simple proof of the Siu-Beauville theorem is due to Catanese [10. Firstly, one notes that II and III each imply the following:

IV. $H^{1}(M ; \mathbb{R})$ admits an isotropic subspace of dimension $\geq 2$.

Here a subspace $U \subset H^{1}(M ; \mathbb{R})$ is said to be isotropic if the cup product map $\Lambda^{2} U \longrightarrow H^{2}(M ; \mathbb{R})$ vanishes identically. Secondly, from an isotropic subspace as in IV, a straightforward application of Hodge theory produces two holomorphic one-forms with trivial wedge product, to which the Castelnuovo-de Franchis lemma can be applied; cf. 10, 11. Thus, on a compact Kähler manifold, conditions I, II, III and IV are all equivalent.

For compact complex surfaces it is known that the Siu-Beauville theorem holds by appealing to the Kodaira classification in the non-Kähler case; see [1, Chapter 2]. It is also known that statement IV is strictly weaker than I and II in this case; the Kodaira-Thurston manifold is a compact complex surface for which IV holds although the surface does not fiber over a curve of genus $\geq 2$.

The main results of this paper are Theorems 3.1 and 3.4. proved in Section 3 , Theorem 3.1 says that conditions I, II and III are equivalent for compact pluriKähler-Weyl manifolds. This unifies the known results for Kähler manifolds and complex surfaces and generalizes them to locally conformally Kähler manifolds with pluricanonical metrics. Our argument applies the strategy of Siu [26] using Weyl harmonic instead of the usual harmonic maps, and it relies on some of the results from [19]. As an immediate corollary of the proof we will see that non-abelian free groups cannot be the fundamental groups of compact pluri-Kähler-Weyl manifolds. For Kähler manifolds this is of course an easy application of Hodge theory together with covering arguments. For compact complex surfaces the corollary was also known as a consequence of classification results; see [1, Chapter 2]. For pluriKähler-Weyl manifolds of higher dimension, the corollary is new. In Theorem 3.4 we prove that statement I holds more generally, assuming only that $\pi_{1}(M)$ admits a representation with non-cyclic image in the fundamental group of some hyperbolic manifold, which does not have to be a surface.

In Section 4 we apply the main results to twistor spaces of (half-)conformally flat manifolds. The conclusion is that if the fundamental group is large, then a twistor space cannot be pluri-Kähler-Weyl. This generalizes various results to the effect that Kählerian twistor spaces are simply connected; cf. [4], [8], [16], 27]. 
In Section 5 we give further applications of Theorem 3.1. We prove that pluriKähler-Weyl manifolds with certain fundamental groups have to be Kähler, and we give obstructions to the existence of pluri-Kähler-Weyl structures on some complex manifolds.

\section{Preliminaries AND BaCKGround}

2.1. Kähler-Weyl geometry. Let $(M, c)$ be a smooth conformal manifold. A Weyl structure or Weyl connection on $(M, c)$ is a torsion-free connection $\nabla^{W}$ which preserves the conformal structure $c$; this means that for any $g \in c$ there exists a 1-form $\theta$ (sometimes called a Higgs field) such that

$$
\nabla^{W} g=\theta \otimes g \text {. }
$$

A standard calculation shows that this condition is equivalent to

$$
\nabla_{X}^{W} Y=\nabla_{X} Y-\frac{1}{2}\left(\theta(X) Y+\theta(Y) X-g(X, Y) \theta^{\sharp}\right),
$$

where $\nabla$ is the Levi-Civita connection of $g$, and $\theta^{\sharp}$ is the vector field $g$-dual to $\theta$. Clearly the space of Weyl connections is a non-empty affine space whose vector space of translations is formed by 1 -forms.

The property of being Hermitian with respect to a fixed almost complex structure $J$ is conformally invariant, and thus the following definition makes sense:

Definition 2.1 ([7]). A Kähler-Weyl structure on $M$ is a triple $\left(J, c, \nabla^{W}\right)$, where $J$ is an almost complex structure, $c$ is a conformal structure which is Hermitian with respect to $J$, and $\nabla^{W}$ is a Weyl connection which preserves $J$; i.e. $\nabla^{W} J=0$.

Since the Weyl connection is torsion-free by definition, the condition $\nabla^{W} J=$ 0 implies that $J$ is integrable. Thus, there is no loss of generality in assuming from the outset that $(M, J)$ is a complex manifold. In the special case when the Weyl connection coincides with the Levi-Civita connection of $g \in c$, the above definition reduces to one of the standard definitions of a Kähler structure. In complex dimension one every conformal structure is part of a Kähler-Weyl structure which is in fact Kähler.

For a given metric $g \in c$ the fundamental two-form $\omega$ of the Hermitian structure $(M, J, g)$ is defined as usual by

$$
\omega(X, Y)=g(X, J Y) .
$$

For a Kähler-Weyl structure the defining conditions imply

$$
d \omega=\omega \wedge \theta,
$$

which means that $\theta$ is the Lee form of the Hermitian structure $(M, J, g)$. Moreover, (2.2) implies

$$
\omega \wedge d \theta=0
$$

In complex dimensions $\geq 3$ the multiplication with the fundamental 2 -form is injective on 2-forms, and we conclude that the Lee form $\theta$ is closed. Therefore in these dimensions any metric $g \in c$ is locally conformally Kähler. In more detail, the form $\theta$ is locally exact by the Poincare lemma, $\theta=d f$. The locally defined metric $e^{-f} g$ is preserved by the Weyl connection $\nabla^{W}$ and, hence, is Kähler. Conversely, given a locally conformally Kähler metric, the Levi-Civita connections of the (essentially unique) locally defined Kähler metrics fit together to form a global Weyl connection. 
In complex dimension $=2$, the identity (2.2) is true (for some $\theta$, uniquely determined by $\omega$ ) for any Hermitian metric, because the multiplication with the fundamental two-form is an isomorphism on 1-forms. The form $\theta$ defines a Weyl connection by formula (2.1), which actually preserves the complex structure.

We summarize this discussion in the following:

Proposition 2.2. In complex dimensions $\leq 2$ every complex manifold admits a Kähler-Weyl structure. In complex dimensions $\geq 3$ a complex manifold admits a Kähler-Weyl structure if and only if it admits a locally conformally Kähler structure.

Let $(M, c)$ be a conformal manifold equipped with a Weyl connection. Recall that by the theorem of Gauduchon [13] there exists a canonical metric $g \in c$, unique up to homothety, such that the corresponding Lee form is co-closed, $\operatorname{tr}_{g}(\nabla \theta)=0$. For example, for a globally conformally Kähler manifold the canonical metric is Kähler and its Lee form vanishes identically. Below we shall consider Kähler-Weyl manifolds whose canonical metrics satisfy an extra hypothesis introduced in [19]:

Definition 2.3 ([19]). A metric $g \in c$ on a Kähler-Weyl manifold is called pluricanonical if the $(1,1)$-part of the covariant derivative of the Lee form vanishes; i.e. $(\nabla \theta)^{1,1}=0$.

Note that all Vaisman manifolds (also called generalized Hopf manifolds) have pluricanonical metrics. These manifolds actually satisfy a much stronger hypothesis: they admit metrics with parallel Lee form; i.e. $\nabla \theta=0$. Vaisman geometry has close links with Sasakian geometry and has been studied intensively in recent years. We refer to [12, 21] for the details and references on this subject.

2.2. Weyl harmonic maps. Let $(M, c)$ be a conformal manifold equipped with a Weyl connection and $(N, h)$ be an arbitrary Riemannian manifold.

For a fixed metric $g \in c$ consider the equation $\operatorname{tr}_{g}(\widetilde{\nabla} D f)=0$ for maps $f: M \longrightarrow$ $N$. Here the differential $D f$ is thought of as a section of $\operatorname{Hom}\left(T M, f^{*} T N\right)=$ $T^{*} M \otimes f^{*} T N$, and $\widetilde{\nabla}$ is defined as the tensor product of the dual Weyl connection with the pullback of the Levi-Civita connection of $(N, h)$. The property of a map to be a solution of this equation does not depend on the choice of a reference metric $g \in c$.

Definition 2.4 ([19]). A map $f: M \longrightarrow N$ from a Weyl manifold $M$ to a Riemannian manifold $N$ is called Weyl harmonic if it solves the equation $\operatorname{tr}_{g}(\widetilde{\nabla} D f)=0$.

In the special case when the Weyl connection is the Levi-Civita connection of $(M, g)$, the defining equation reduces to the harmonic map equation. In general, it differs from the usual harmonic map equation by the term $((n-2) / 2) D f\left(\theta^{\sharp}\right)$, where $n$ is the real dimension of $M$. Thus, for two-dimensional domains the Weyl harmonic maps are precisely the usual harmonic maps.

We refer to [19] for basic existence and uniqueness results for Weyl harmonic maps. An important ingredient for our arguments here concerns pluriharmonicity of Weyl harmonic maps. This occurs when the Kähler-Weyl domain $M$ has complex dimension 2 or admits a pluricanonical metric; see [19, Theorem 4.2]. This motivates the following:

Definition 2.5. A Kähler-Weyl manifold is called pluri-Kähler-Weyl if it is twodimensional or if it admits a pluricanonical metric in the sense of Definition 2.3 . 
Another important ingredient in our proofs will be the following strengthened version of results of Carlson-Toledo [9] and Jost-Yau [17]:

Theorem 2.6 ([19]). Let $M$ be a closed complex manifold and $N$ a Riemannian manifold of constant negative curvature. If $f: M \longrightarrow N$ is a pluriharmonic map whose rank is at most two and equals two on an open and dense subset of $M$, then there exists a compact Riemann surface $S$, a holomorphic map $h: M \longrightarrow S$ and a harmonic map $\phi: S \longrightarrow N$ such that $f=\phi \circ h$.

\section{Fibrations over CURVES}

We now prove the following generalization of Theorem 1.1

Theorem 3.1. Let $M$ be a closed complex manifold admitting a pluri-Kähler-Weyl structure. Then the following three statements are equivalent:

I. $M$ admits a surjective holomorphic map with connected fibers to a closed Riemann surface of genus $\geq 2$,

II. the fundamental group $\pi_{1}(M)$ admits a surjective homomorphism to the fundamental group of a closed Riemann surface of genus $\geq 2$, and

III. the fundamental group $\pi_{1}(M)$ admits a surjective homomorphism to a nonabelian free group.

Proof. As noted in the Introduction, each of the statements implies the next one. Thus, we have to prove only that III implies I. Clearly we may assume that the complex dimension of $M$ is at least 2. Fix a pluri-Kähler-Weyl structure on $M$.

Suppose $\pi_{1}(M)$ surjects onto $F_{k}$, the free group of rank $k \geq 2$. We can compose this surjection with the homomorphism $F_{k} \longrightarrow \pi_{1}\left(\Sigma_{k}\right)$ given by sending the $i$ th standard generator of $F_{k}$ to a standard generator of the fundamental group of the $i$ th summand in a decomposition of $\Sigma_{k}$ as a connected sum of $k$ tori. This gives us a homomorphism $\varphi: \pi_{1}(M) \longrightarrow \pi_{1}\left(\Sigma_{k}\right)$ whose image is a non-abelian free group; in particular it is not cyclic. Let $f: M \longrightarrow \Sigma_{k}$ be a smooth map with $f_{*}=\varphi$ on $\pi_{1}(M)$.

Now choose a hyperbolic metric on $\Sigma_{k}$. Since the image of $\varphi$ is not cyclic, by [19, Theorem 2.2] the map $f$ is homotopic to a Weyl harmonic map, which we also denote by $f$. By unique continuation, the latter map cannot be constant on an open set. Further, it has rank two on an open and dense subset of $M$, because otherwise by [19, Proposition 1.2] its image would be a closed geodesic, contradicting the fact that the image of $f_{*}$ is not cyclic.

Recall that the hyperbolic metric on $\Sigma_{k}$ has non-positive Hermitian sectional curvature in the sense of Sampson; cf. [1, Chapter 6]. Therefore, by [19, Theorem 4.2] any Weyl harmonic map is pluriharmonic. To summarize, we have a pluriharmonic map $f: M \longrightarrow \Sigma_{k}$ inducing $\varphi: \pi_{1}(M) \longrightarrow \pi_{1}\left(\Sigma_{k}\right)$, and the rank of the differential $D f$ is two on an open and dense subset of $M$. Thus we can apply the factorization theorem, Theorem 2.6 to conclude that $f$ factors through a holomorphic map $h: M \longrightarrow S$ to a compact Riemann surface. Clearly, the genus of $S$ is at least two, $g(S) \geq 2$.

Consider the Stein factorization of the map $h$,

$$
M \stackrel{h_{1}}{\longrightarrow} C \stackrel{h_{2}}{\longrightarrow} S,
$$

where $h_{1}$ has connected fibers. Then $g(C) \geq g(S) \geq 2$, and $h_{1}$ has to be non-trivial on $\pi_{1}(M)$. Since the map $h_{1}$ is holomorphic and $M$ is compact, we conclude that 
$h_{1}$ is surjective. Thus the map $h_{1}$ satisfies all the requirements in statement I, and the theorem is proved.

Remark 3.2. Suppose $k$ is the maximal integer for which $\pi_{1}(M)$ surjects onto $F_{k}$. (A maximal $k$ exists and is bounded above by $b_{1}(M)$.) Then the genus of $C$ cannot be larger than $k$, because $\left(h_{1}\right)_{*}$ is surjective on $\pi_{1}(M)$ and $\pi_{1}\left(\Sigma_{g}\right)$ surjects onto $F_{g}$. The above proof then shows that the genus of $C$ is in fact equal to $k$.

Corollary 3.3. A non-abelian free group cannot be the fundamental group of a closed pluri-Kähler-Weyl manifold.

Proof. Suppose that $M$ is a pluri-Kähler-Weyl manifold whose fundamental group $\pi_{1}(M)$ is isomorphic to $F_{k}$, where $k \geq 2$. Fix an isomorphism $\varphi: \pi_{1}(M) \longrightarrow$ $F_{k}$. The proof of Theorem 3.1 shows that $\varphi$ factors through a surjection to the fundamental group of a closed Riemann surface and thus cannot be injective, a contradiction.

Replacing the hyperbolic surfaces in Theorem 3.1 by closed hyperbolic manifolds of higher dimension, we obtain the following generalization:

Theorem 3.4. Let $M$ be a closed complex manifold admitting a pluri-KählerWeyl structure, and let $N$ be a closed Riemannian manifold of constant negative curvature. If $\varphi: \pi_{1}(M) \longrightarrow \pi_{1}(N)$ is a representation with non-cyclic image, then there exists a compact Riemann surface $S$ and a holomorphic map $h: M \longrightarrow S$ with connected fibers such that $\varphi$ factors through $h_{*}$.

Note that under these hypotheses statements I, II and III in Theorem 3.1 hold for $M$. The proof of Theorem 3.4 is similar to that of Theorem 3.1 cf. also the proof of [19, Theorem 5.5], where the situation when $\varphi$ is an isomorphism is considered. In slightly more detail, there is a Weyl harmonic map from $M$ equipped with a pluri-Kähler-Weyl structure to $N$ with its hyperbolic metric which induces $\varphi$ on $\pi_{1}(M)$. This map is pluriharmonic and has rank at least two on an open and dense subset of $M$, just as in the above proof of Theorem 3.1. By [19, Corollary 4.6], the rank is at most two. Therefore, the conclusion follows using Theorem 2.6.

As an immediate consequence of Theorem 3.4 we have the following result, which is interesting in the context of the relation $M \geq N$ on manifolds defined by the existence of maps $M \longrightarrow N$ of non-zero degree. We refer to [9], 20, for further information on this relation.

Corollary 3.5. Let $M$ be a closed pluri-Kähler-Weyl manifold, and let $N$ be a closed real hyperbolic manifold of dimension $\geq 4$. Then every map $f: M \longrightarrow N$ has degree zero.

Proof. If the degree were non-zero, then $f_{*}: \pi_{1}(M) \longrightarrow \pi_{1}(N)$ would be surjective onto a finite index subgroup. By Theorem 3.4 the harmonic map in the homotopy class of $f$ would factor through a two-dimensional manifold, showing that the degree had to be zero after all.

In [19, Theorem 5.5], it was proved that the fundamental groups of closed hyperbolic manifolds of dimension $\geq 3$ cannot be fundamental groups of closed pluriKähler-Weyl manifolds. We now extend this result: 
Corollary 3.6. Let $N$ be a closed hyperbolic manifold of dimension $\geq 3$. Any group $\Gamma$ which fits into a central extension of the form

$$
1 \longrightarrow \mathbb{Z}^{k} \longrightarrow \Gamma \stackrel{\varphi}{\longrightarrow} \pi_{1}(N) \longrightarrow 1
$$

cannot be the fundamental group of a closed pluri-Kähler-Weyl manifold.

Proof. Let $M$ be a Kähler-Weyl manifold whose fundamental group is $\Gamma$. Then, by Theorem 3.4. $\varphi$ factors through a surjective homomorphism $f_{*}: \Gamma \longrightarrow \pi_{1}(S)$, for some closed Riemann surface $S$ of genus $\geq 2$. Since $\pi_{1}(S)$ has trivial center, the surjection $f_{*}$ descends from $\Gamma$ to the quotient $\pi_{1}(N)$. However, the identity of $\pi_{1}(N)$ cannot factor through $\pi_{1}(S)$, and we obtain a contradiction.

Note that the dimension assumption on $N$ cannot be dropped, as the direct product of $\mathbb{Z}^{2}$ with any surface group is the fundamental group of a compact complex, in fact Kähler, surface.

Example 3.7. Let $N$ be a closed hyperbolic 3-manifold, and let $M \longrightarrow N$ be a circle bundle. Then the total space $M$ does not admit a complex structure by Corollary 3.6. A weaker statement in this direction is proved in [1] using the Enriques-Kodaira classification.

\section{TWISTOR SPACES}

An oriented Riemannian four-manifold is called half-conformally flat if its Weyl tensor is either self-dual or anti-self-dual. This is a conformally invariant condition. By work of Penrose and Atiyah-Hitchin-Singer [2, a half-conformally flat fourmanifold $N$ has associated to it a complex three-fold $Z$, called its twistor space, which is differentiably a two-sphere bundle over $N$. In particular it has the same fundamental group as $N$. It is a theorem of Taubes [28] that every closed oriented four-manifold admits a metric with anti-self-dual Weyl tensor after stabilization by connected summing with many copies of $\overline{\mathbb{C} P^{2}}$. In particular every finitely presentable group is the fundamental group of a compact complex three-fold obtained as the twistor space of a suitable four-manifold.

Recall that a discrete group is called large if it has a finite index subgroup that admits a surjective homomorphism to $F_{2}$. This notion was introduced by Gromov [15] and has many important ramifications, for example in geometric group theory and in spectral geometry. For twistor spaces we have:

Theorem 4.1. Let $N$ be a closed half-conformally flat four-manifold with large fundamental group. Then its twistor space is a complex manifold that does not admit any pluri-Kähler-Weyl structure.

This should be compared with a result of Hitchin [16, who showed that the only Kähler twistor spaces are those of $S^{4}$ and of $\overline{\mathbb{C} P^{2}}$; in particular, Kähler twistor spaces are simply connected. Theorem 4.1 shows that under the weaker pluriKähler-Weyl assumption we can still conclude that the fundamental group is not large.

Proof. The assumption about the fundamental group means that after replacing $N$ by some finite covering, its fundamental group surjects to $F_{2}$. As finite covers of pluri-Kähler-Weyl manifolds are pluri-Kähler-Weyl, and the twistor space has the same fundamental group as the four-manifold, we may assume for a contradiction 
that we have a twistor space $Z$ whose fundamental group surjects to $F_{2}$. By Theorem 3.1 this implies that $Z$ fibers holomorphically with connected fibers over a curve $C$ of genus $\geq 2$. The pullback by $h: Z \longrightarrow C$ is injective on $H^{1}(C ; \mathbb{C})$. This cohomology group has a Hodge decomposition, and so we obtain a contradiction as soon as we see that $Z$ has no holomorphic one-forms. But this is a standard fact; compare [1, p. 27] or Lemma 4.4 below.

Remark 4.2. Sometimes $N$ can be chosen as a complex Kähler surface with antiself-dual Weyl tensor, e.g. $\mathbb{C} P^{1} \times C$, for a curve $C$ of genus $\geq 2$. In these cases the twistor space $Z$ is diffeomorphic to $\mathbb{P}\left(\mathcal{O}_{N} \oplus \mathcal{O}_{N}\left(K_{N}\right)\right)$, where $K_{N}$ is the canonical bundle of $N$. This shows that the twistor complex structure is not pluri-KählerWeyl although the smooth manifold underlying $Z$ also carries a Kähler complex structure.

Example 4.3. There are complex algebraic surfaces $N$ which are ball quotients $\mathbb{C} H^{2} / \Gamma$, and which fiber holomorphically over curves of genus $\geq 2$; cf. for example 3]. In particular, the latter property implies that they have large fundamental groups. Since the Bergmann metric on $\mathbb{C} H^{2}$ has self-dual Weyl tensor, the twistor space $Z$ of such an $N$ is a complex manifold. By Theorem 4.1, it cannot admit a pluri-Kähler-Weyl structure.

We shall show in Corollary 5.4 below that the largeness of the fundamental group can be dispensed with in this example.

There is a well known generalization of the construction of twistor spaces for oriented conformally flat manifolds of arbitrary even dimension $2 n$. We briefly recall this construction, referring to [24, [27, , 4] for further details.

Let $(N, h)$ be an oriented Riemannian $2 n$-manifold, and let $Z$ be the quotient of its oriented orthonormal frame bundle by $U(n) \subset S O(2 n)$. This is the bundle of pointwise orthogonal complex structures on $N$ compatible with the orientation. The total space $Z$, called the twistor space of $N$, carries a tautological almost complex structure, which is integrable if $h$ is conformally flat. In this case $Z$ is a complex manifold, and the fibers of the projection $Z \longrightarrow N$ are holomorphic submanifolds isomorphic to the Hermitian symmetric space $X_{n}=S O(2 n) / U(n)$. For example, if $N=S^{2 n}$ is a round sphere, the twistor space is $X_{n+1}$, with the fiber $X_{n}$ embedded in the standard way.

Lemma 4.4. Let $Z$ be the twistor space of a conformally flat manifold $N$. Then $Z$ has no non-trivial holomorphic one-forms.

Proof. The fibers of the projection $\pi: Z \longrightarrow N$ sweep out $Z$, so it is enough to show that for a fiber $F$ the restriction $\left.\Omega_{Z}^{1}\right|_{F}$ has no non-trivial holomorphic sections. Taking a conformal chart for $N$ around $\pi(F)$, we can identify $\left.\Omega_{Z}^{1}\right|_{F}$ with $\left.\Omega_{X_{n+1}}^{1}\right|_{X_{n}}$. The natural Kähler-Einstein metric of $X_{n+1}$ of positive Ricci curvature induces a metric of negative mean curvature on this bundle, which therefore has no holomorphic sections by a standard application of the Bochner vanishing argument; cf. [18, Chapter III].

Combining Theorem 3.1 and Lemma 4.4, we conclude:

Theorem 4.5. The twistor space of a closed conformally flat manifold with large fundamental group is a complex manifold which is not pluri-Kähler-Weyl. 
This generalizes the results of [27, [8], 4], showing that Kählerian twistor spaces are simply connected. There are many conformally flat manifolds to which Theorem 4.5 applies. For example, it is well known that connected sums of conformally flat manifolds are again conformally flat. Thus, given any two conformally flat manifolds of the same dimension with positive first Betti numbers, their connected sum satisfies the hypotheses of the theorem.

For some conformally flat manifolds, more can be said than in Theorem 4.5 , not only is the twistor space not pluri-Kähler-Weyl, but in fact no manifold with the same fundamental group is pluri-Kähler-Weyl. This is so for the conformally flat manifolds $\left(S^{1} \times S^{2 n-1}\right) \# \ldots \#\left(S^{1} \times S^{2 n-1}\right)$ by Corollary 3.3 , and for real hyperbolic manifolds by [19, Theorem 5.5]. Here are some more examples:

Example 4.6. Let $N$ be any closed oriented real hyperbolic manifold of dimension $2 n-1 \geq 3$. Then $N \times S^{1}$ with the product metric is conformally flat. Its twistor space $Z$ has fundamental group $\pi_{1}(N) \times \mathbb{Z}$, and no manifold with such a fundamental group can be pluri-Kähler-Weyl by Corollary 3.6. Some hyperbolic manifolds also have non-trivial circle bundles over them whose total spaces are conformally flat (see [5]), and Corollary [3.6 applies to their fundamental groups as well.

Remark 4.7. There is a notion of twistor spaces for quaternionic Kähler manifolds in the sense of Salamon 23. Our discussion could be generalized to this case, but the generalization would be vacuous. Quaternionic Kähler manifolds are always Einstein, and so have constant scalar curvature. If the scalar curvature is positive, then Salamon [23] proved that the twistor space is Kähler and simply connected. If the scalar curvature is negative, Semmelmann and Weingart 25] proved that the first Betti number vanishes, and so every locally conformally Kähler structure on the twistor space would in fact be Kähler. In the Kähler case, Campana [8] showed that the fundamental group is trivial. Finally, in the case of zero scalar curvature, the Cheeger-Gromoll splitting theorem shows that the fundamental group is virtually abelian and therefore not large.

\section{Further APPLiCATIONS}

5.1. When Kähler-Weyl implies Kähler. Vaisman has put forward the philosophy that Kähler-Weyl manifolds which are, in a suitable sense, topologically Kähler should in fact be Kähler; cf. [29, 12]. In this direction, he proved the following:

Proposition 5.1 (29]). A compact locally conformally Kähler manifold which admits some Kähler metric, or, more generally, which satisfies the $\partial \bar{\partial}$-Lemma, is globally conformally Kähler.

We shall give a proof, following [12, because we need the argument for our next result.

Proof. Suppose $(M, g, J, \omega)$ is locally conformally Kähler of complex dimension $n$, with Lee form $\theta$. Let $\alpha=\theta \circ J$ be the anti-Lee form. The definition of the Lee form implies that

$$
\alpha=-\frac{1}{n-1} d_{g}^{*} \omega
$$

where $d_{g}^{*}$ is the formal $L^{2}$-adjoint of the exterior derivative with respect to $g$; compare [12]. If we can globally conformally rescale the metric so that for the new 
metric the corresponding $\alpha$ is closed, then $\alpha$ is closed and co-closed and therefore harmonic. However, it is also in the image of $d^{*}$, and so the Hodge decomposition theorem implies that $\alpha$ vanishes. This means that the Lee form vanishes, and so the new metric is Kähler.

We know that $\theta$ is always a closed real one-form. Decomposing $d \theta$ into $(p, q)$ types, we find that $\partial \theta^{1,0}=0=\bar{\partial} \theta^{0,1}$ and $\partial \theta^{0,1}+\bar{\partial} \theta^{1,0}=0$.

The anti-Lee form is $\alpha=i\left(\theta^{1,0}-\theta^{0,1}\right)$. Now the above relations obtained from $d \theta=0$ imply that $d \alpha=2 i \bar{\partial} \theta^{1,0}$. Thus $d \alpha$ is an exact form of pure type $(1,1)$. Therefore the $\partial \bar{\partial}$-Lemma implies that there is a globally defined real function $\varphi$ on $M$ such that $d \alpha=2 i \partial \bar{\partial} \varphi$.

Consider the metric $h=\exp (\varphi) g$. This is locally conformally Kähler with fundamental two-form $\exp (\varphi) \omega$ and Lee form $\theta+d \varphi$. Its anti-Lee form is $\alpha+i(\partial \varphi-\bar{\partial} \varphi)$, which is closed because $d \alpha=2 i \partial \bar{\partial} \varphi$. This completes the proof.

We now extend Vaisman's result in the following way:

Proposition 5.2. Let $M$ be a closed locally conformally Kähler manifold admitting a holomorphic map $f: M \longrightarrow N$ to a complex manifold $N$ which is Kähler or which at least satisfies the $\partial \bar{\partial}$-Lemma. Assume that $f^{*}: H^{1}(N ; \mathbb{R}) \longrightarrow H^{1}(M ; \mathbb{R})$ is an isomorphism. Then $M$ is globally conformally Kähler.

Proof. Since $f^{*}$ is an isomorphism, the cohomology class of the Lee form $\theta$ is the pullback of some class on $N$. After rescaling the given locally conformally Kähler metric on $M$, we may assume that the Lee form $\theta$ is itself the pullback of a closed form $\beta$ on $N$. As $f$ is holomorphic and $f^{*} \beta=\theta$, we find that the anti-Lee form $\alpha=\theta \circ J$ equals $f^{*}(\beta \circ J)$, where we also use $J$ to denote the complex structure on $N$, not just on $M$. As in the previous proof, the $\partial \bar{\partial}$-Lemma, applied now on $N$, not on $M$, tells us that there is a real-valued function $\varphi$ on $N$ such that $d(\beta \circ J)=2 i \partial \bar{\partial} \varphi$. Pulling back to $M$ we find $d \alpha=2 i \partial \bar{\partial}(\varphi \circ f)$. As in the previous proof, the metric $\exp (\varphi \circ f) g$ on $M$ is Kähler, because its Lee form vanishes identically.

This proposition allows us to prove the following:

Theorem 5.3. Let $M$ be a closed pluri-Kähler-Weyl manifold whose fundamental group admits a surjection $\varphi: \pi_{1}(M) \longrightarrow \pi_{1}\left(\Sigma_{g}\right)$ with $g \geq 2$ for which $\varphi^{*}: H^{1}\left(\Sigma_{g} ; \mathbb{R}\right)$ $\longrightarrow H^{1}(M ; \mathbb{R})$ is an isomorphism. Then $M$ admits a Kähler metric.

Proof. First suppose that $M$ is a complex surface. Since $f^{*}$ is an isomorphism on the first cohomology, $M$ has even first Betti number because $N$ does. A compact complex surface with even first Betti number is Kähler; see Buchdahl [6] for a proof that is independent of the Kodaira classification.

If the complex dimension of $M$ is at least 3 , then we apply Theorem 3.1 and Remark 3.2 to obtain a holomorphic map to a closed complex curve inducing an isomorphism in the first cohomology. In this case $M$ is locally conformally Kähler, and applying Proposition 5.2 to the fibration over a curve shows that it is in fact globally conformally Kähler.

We can also apply Proposition 5.2 to the twistor spaces of ball quotients to obtain the following generalization of Example 4.3

Corollary 5.4. Let $N=\mathbb{C} H^{2} / \Gamma$ be a compact ball quotient. Then its twistor space $Z$ is a complex manifold which is not pluri-Kähler-Weyl. 
Proof. Consider the projection $\pi: Z \longrightarrow N$. Its fibers are two-spheres, and so the Euler class of the vertical tangent bundle does not vanish when evaluated on a fiber. Thus the fibers are not null-homologous, and any smooth map homotopic to $\pi$ must have maximal rank somewhere.

Now suppose $Z$ is pluri-Kähler-Weyl. Then $\pi$ is homotopic to a Weyl harmonic map by the results of [19. As the rank of the Weyl harmonic representative in the homotopy class of $\pi$ is greater than 2, it is holomorphic by [19, Corollary 4.6]. Now we can apply Proposition 5.2 to this holomorphic map to conclude that $Z$ is Kähler. But this contradicts the results of [16], 8].

5.2. Period domains. Denote by $X$ the symmetric space $S O(2 p, q) /(S O(2 p) \times$ $S O(q)$ ); it is Hermitian symmetric if and only if $p=1$ or $q=2$. By the general construction in [14, the space $X$ has associated to it a homogeneous complex manifold $D=S O(2 p, q) /(U(p) \times S O(q))$, which one can think of as a twistor space; here the isotropy group of $D$ is the centralizer of a torus in $S O(2 p) \times S O(q)$. The manifold $D$ parameterizes Hodge structures of weight 2 and is an example of a Griffiths period domain; see [14], 1] and the references therein for the details.

The following theorem generalizes an analogous result of Carlson and Toledo in the Kähler setting; cf. [1, Chapter 6].

Theorem 5.5. Let $\Gamma$ be a torsion-free cocompact lattice in $S O(2 p, q)$, where $p>1$ and $q>2$. Then the compact complex manifold $D / \Gamma$ is not homotopy equivalent to a compact pluri-Kähler-Weyl manifold.

Proof. Let $\pi: D \longrightarrow X$ be the natural topologically trivial fibration with fiber $X_{p}=S O(2 p) / U(p)$. We denote by $c_{1}$ the Chern class of the anti-canonical bundle of $D / \Gamma$ and let $\Omega$ be a volume form on $X / \Gamma$. Since the fiber $X_{p}$ is a compact Hermitian symmetric space of positive Ricci curvature, its anti-canonical bundle is positive; thus $c_{1}^{r}>0$ on $X_{p}$, where $r$ is the complex dimension of $X_{p}$. By the Fubini theorem

$$
\int_{D / \Gamma} \pi^{*} \Omega \wedge c_{1}^{r}>0
$$

and, therefore, the cohomology class of $\pi^{*} \Omega$ is non-zero. Suppose there exists a compact pluri-Kähler-Weyl manifold $M$ and a homotopy equivalence $f: M \longrightarrow$ $D / \Gamma$. Then the map $g=\pi \circ f: M \longrightarrow X / \Gamma$ is non-trivial in top-degree cohomology, contradicting [19, Corollary 5.2].

Remark 5.6. It is not hard to see that the natural complex structure on $D / \Gamma$ is not Kähler. This follows from the known fact that the deformation space of a fiber of $\pi$ is not compact. However, the generalization to the class of pluri-Kähler-Weyl manifolds is already non-trivial. The point of the theorem is, of course, that no complex structure on a homotopy equivalent manifold can be pluri-Kähler-Weyl.

Remark 5.7. Theorem 5.5 applies to all the locally homogeneous complex manifolds studied by Griffiths and Schmid [14] as long as $X$ is not Hermitian symmetric and $X / \Gamma$ has non-zero Euler number. The condition on the Euler number is required for the applicability of the results of [19].

\section{POSTSCRIPT}

The original motivation for this work was the extension of topological restrictions on Kähler manifolds to all Kähler-Weyl manifolds. It is clear that some such 
extensions are possible, as shown by the fact that, in complex dimension $\geq 3$, Kähler-Weyl manifolds are locally conformally Kähler and, if the first Betti number vanishes, globally conformally Kähler and therefore Kählerian. This leads to:

Example 6.1. Let $\Gamma$ be a finitely presentable group with $b_{1}(\Gamma)=0$ that is not a Kähler group. Then $\Gamma$ cannot be the fundamental group of any Kähler-Weyl manifold.

We do not have to worry about complex dimension two in this example, because in that dimension the vanishing of the first Betti number implies that a complex manifold is Kähler.

Example 6.1 applies in many concrete instances, e.g. when $\Gamma$ is the fundamental group of a hyperbolic homology sphere or when it is a free product of two nontrivial groups with vanishing first Betti number; compare 11. Thus these groups are not fundamental groups of Kähler-Weyl or locally conformally Kähler manifolds in any dimension. Recall however that, by the result of Taubes [28], every finitely presentable group is the fundamental group of a compact complex three-fold.

There are also restrictions that apply to specific complex structures only rather than to all complex structures on a given manifold.

Example 6.2. Let $M$ be a compact complex manifold that admits a holomorphic map $f: M \longrightarrow B$ to a Kähler manifold $B$ for which $f^{*}$ is an isomorphism on degreeone cohomology with real coefficients, and some smooth fiber $F=f^{-1}(p)$ is not Kählerian. Then $M$ cannot be locally conformally Kähler.

The assumption about $f^{*}$ implies, via Proposition 5.2. that if $M$ is locally conformally Kähler, then it is Kählerian. In this case, the complex submanifold $F \subset M$ also has to be Kählerian, leading to a contradiction.

For technical reasons, which stem from the work in [19], in this paper we have proved the Siu-Beauville theorem only for pluri-Kähler-Weyl rather than for all Kähler-Weyl manifolds. In fact the stronger assumption is needed for only one step in the proof, to conclude that Weyl harmonic maps are pluriharmonic. It remains an open problem to decide whether the result is true for all Kähler-Weyl manifolds.

After this paper was written, Ornea and Verbitsky 22 pointed out that pluriKähler-Weyl manifolds are topologically Vaisman and that this point of view gives alternative proofs of some of the corollaries of Theorem 3.1

In summary, there are many compact complex manifolds that are not KählerWeyl, and there are also Kähler-Weyl manifolds that are not pluri-Kähler-Weyl.

\section{ACKNOWLEDGEMENTS}

The first author was supported by an EPSRC fellowship at the University of Edinburgh. Parts of this work were done while the second author was taking part in the activity on "Extremal Kähler metrics and Kähler-Ricci flow" at the Centro Ennio De Giorgi in Pisa. He is grateful to S. Salamon for the invitation and for a helpful suggestion in connection with this work. The paper was completed while the second author enjoyed the support of The Bell Companies Fellowship at the Institute for Advanced Study in Princeton. 


\section{REFERENCES}

1. J. Amorós, M. Burger, K. Corlette, D. Kotschick and D. Toledo, Fundamental Groups of Compact Kähler Manifolds, Math. Surveys Monogr., 44, Amer. Math. Soc., Providence, RI, 1996. MR.1379330 (97d:32037)

2. M. F. Atiyah, N. J. Hitchin and I. Singer, Self-duality in four-dimensional Riemannian geometry, Proc. Royal Soc. London Ser. A 362 (1978), 425-461. MR506229 (80d:53023)

3. G. Barthel, F. Hirzebruch und T. Höfer, Geradenkonfigurationen und Algebraische Flächen, Vieweg Verlag, 1987. MR912097(89a:14045)

4. P. de Bartolomeis, L. Migliorini and A. Nannicini, Propriétés globales de l'espace de twisteurs, Atti Accad. Naz. Lincei Cl. Sci. Fis. Mat. Natur. Rend. Lincei (9) Mat. Appl. 2 (1991), no. 2, 147-153. MR1120134 (92m:53112)

5. I. Belegradek, Some curious Kleinian groups and hyperbolic 5-manifolds, Transf. Groups 2 (1997), 3-29. MR.1439244 (98d:57066)

6. N. Buchdahl, On compact Kähler surfaces, Ann. Inst. Fourier (Grenoble) 49 (1999), 287-302. MR 1688136 (2000f:32029)

7. D. M. J. Calderbank and H. Pedersen, Einstein-Weyl geometry, in Essays on Einstein Manifolds, Surveys in Differential Geometry, VI, Int. Press, Boston, MA, 1999. MR 1798617 (2002b:53062)

8. F. Campana, Espaces de twisteurs dont l'espace des cycles a ses composantes irréductibles compactes, C. R. Acad. Sci. Paris, Ser. I Math. 308 (1989), 565-568. MR.999456 (90e:32022)

9. J. A. Carlson and D. Toledo, Harmonic mapping of Kähler manifolds to locally symmetric spaces, Publ. Math. I.H.E.S. 69 (1989), 173-201. MR1019964 (91c:58032)

10. F. Catanese, Moduli and classification of irregular Kaehler manifolds (and algebraic varieties) with Albanese general type fibrations, Invent. Math. 104 (1991), 263-289. MR1098610 (92f:32049)

11. G. Deschamps, 4-variétés parallélisables sans structure complexe dont l'espace twistoriel est complexe, C. R. Acad. Sci. Paris, Ser. I 341 (2005), 35-38. MR2153389 (2006e:53089)

12. S. Dragomir and L. Ornea, Locally Conformal Kähler Geometry, Birkhäuser Boston, Boston, MA, 1998. MR1481969 (99a:53081)

13. P. Gauduchon, Le théorème de l'excentricité nulle, C. R. Acad. Sci. Paris, Ser. A-B 285 (1977), A387-A390. MR0470920 (57:10664)

14. P. Griffiths and W. Schmid, Locally homogeneous complex manifolds, Acta Math. 123 (1969), 253-302. MR0259958 (41:4587)

15. M. Gromov, Volume and bounded cohomology, Publ. Math. I.H.E.S. 56 (1982), 5-99. MR686042 (84h:53053)

16. N. J. Hitchin, Kählerian twistor spaces, Proc. London Math. Soc. (3) 43 (1981), 133-150. MR623721 (84b:32014)

17. J. Jost and S.-T. Yau, Harmonic maps and group representations, in Differential Geometry, Pitman Monogr. Surveys Pure Appl. Math., 52, Longman Sci. Tech., Harlow, 1991. MR:1173045 (93g:58036)

18. S. Kobayashi, Differential Geometry of Complex Vector Bundles, Iwanami Shoten Publishers and Princeton Univ. Press, 1987. MR909698 (89e:53100)

19. G. Kokarev, On pseudo-harmonic maps in conformal geometry, Proc. London Math. Soc. (3) 99 (2009), 168-194. MR2520354

20. D. Kotschick and C. Löh, Fundamental classes not representable by products, J. London Math. Soc. (2) 79 (2009), 545-561. MR2506686

21. L. Ornea, Locally conformally Kähler manifolds. A selection of results, Lect. Notes Semin. Interdiscip. Mat., IV, 121-152, S.I.M. Dep. Mat. Univ. Basilicata, Potenza, 2005. MR2222543 (2007c:53101)

22. L. Ornea and M. Verbitsky, Topology of locally conformally Kähler manifolds with potential, preprint, arXiv:0904.3362 v2 [math.DG], 2 May 2009.

23. S. M. Salamon, Quaternionic Kähler manifolds, Invent. Math. 67 (1982), 143-171. MR664330 (83k:53054)

24. S. M. Salamon, Harmonic and holomorphic maps, in Geometry Seminar "Luigi Bianchi" II1984, Lecture Notes in Mathematics, 1164, Springer, Berlin, 1985. MR829230 (88b:58039)

25. U. Semmelmann and G. Weingart, Vanishing theorems for quaternionic Kähler manifolds, J. Reine Angew. Math. 544 (2002), 111-132. MR1887892(2003f:53077) 
26. Y. T. Siu, Strong rigidity for Kähler manifolds and the construction of bounded holomorphic functions, in Discrete Groups in Geometry and Analysis, ed. R. Howe, Birkhäuser Boston, Boston, MA, 1987. MR900825 (89i:32044)

27. M. Slupinski, Espaces de twisteurs Kählériens en dimension $4 k, k>1$, J. London Math. Soc. (2) 33 (1986), 535-542. MR 850969 (87k:53164)

28. C. H. Taubes, The existence of anti-self-dual conformal structures, J. Differential Geometry 36 (1992), 163-253. MR1168984 (93j:53063)

29. I. Vaisman, On locally and globally conformal Kähler manifolds, Trans. Amer. Math. Soc. 262 (1980), 533-542. MR586733 (81j:53064)

School of Mathematics, The University of Edinburgh, King's Buildings, Mayfield RoAd, Edinburgh EH9 3JZ, United Kingdom

E-mail address: G.Kokarev@ed.ac.uk

Current address: Mathematisches Institut, Ludwig-Maximilians-Universität München, Theresienstr. 39, 80333 München, Germany

E-mail address: Gerasim.Kokarev@mathematik.uni-muenchen.de

Mathematisches Institut, Ludwig-Maximilians-Universität München, Theresienstr. 39, 80333 München, Germany

E-mail address: dieter@member.ams.org 